\title{
Nyctalopia and Conjunctival Xerosis Indicating Vitamin A Deficiency in Cystic Fibrosis
}

\author{
M. A. NEUGEBAUER, ${ }^{1}$ S. A. VERNON,${ }^{1}$ G. BRIMLOW,${ }^{2}$ J. C. TYRRELL,${ }^{3}$ \\ E. J. HILLER ${ }^{3}$ and C. MARENAH ${ }^{4}$ \\ Nottingham
}

\begin{abstract}
Summary
Thirty-one Cystic Fibrosis patients were investigated for clinical and biochemical evidence of Vitamin A deficiency. All had been prescribed oral pancreatic enzyme replacements and twice the recommended daily requirement of Vitamin $\mathrm{A}(5000 \mathrm{IU})$. None were aware of any ocular symptoms, but 3 out of 31 ( 10 per cent) were found to have frank conjunctival xerosis and six ( 19 per cent) to have abnormal dark adaptation. There was no correlation between the above findings and abnormal liver function or clinical disease severity. All patients with cystic fibrosis should have regular Vitamin A estimations with ophthalmological assessment if serum levels fall below $30 \mathrm{microgm} / \mathrm{dl}$.
\end{abstract}

Cystic Fibrosis (CF), is an autosomal recessive disorder where there is a generalised dysfunction of exocrine glands. It is a major cause of severe paediatric lung disease and pancreatic insufficiency. There were approximately $5,000 \mathrm{CF}$ patients in the United Kingdom, of whom 25 per cent are adults. ${ }^{1}$ Vitamin $\mathrm{A}$ is essential for the normal differentiation of epithelial cell membranes. ${ }^{2}$ One of the earliest descriptions of $\mathrm{CF}$ detailed xerosis and squamous metaplasia of lung epithelium due to associated Vitamin A deficiency. ${ }^{3}$

Cystic Fibrosis patients in Nottingham, as in many other centres, are now treated with oral pancreatic enzyme replacements, (dosage is varied to control digestive symptoms), and are also prescribed 5000IU of Vitamin A daily, twice the recommended requirement. Such supplementation has contributed to the increased life expectancy of CF patients and reports of ophthalmic manifestations of Vitamin A deficiency have subsequently been rare. ${ }^{4,5,6,7}$

A study of patients attending the Nottingham CF clinic was undertaken to determine the incidence of clinical Vitamin A deficiency in a group of patients who had been prescribed 'adequate' supplementation.

\section{Method}

All patients over the age of twelve, who lived within a reasonable travelling distance were invited to attend for ophthalmological assessment which included slit lamp examination, fundoscopy, and testing of dark adaptation. Two systems were used for this: a Friedmann Field Analyser Mk.1. and an in house computer system, (Fig. 1). The Friedmann analyser was used in the recommended fashion, utilising an auxillary filter bar. ${ }^{8}$ The com-

From 'Department of Ophthalmology, University Hospital, Nottingham; 2Department of Medical Physics, University Hospital, Nottingham; ${ }^{3}$ Department of Paediatrics, City Hospital, Nottingham; ${ }^{4}$ Department of Clinical Biochemistry, City Hospital, Nottingham.

Correspondence to: Mr S. A. Vernon, Senior Lecturer, Department of Ophthalmology, University Hospital, Nottingham NG7 2UH. 


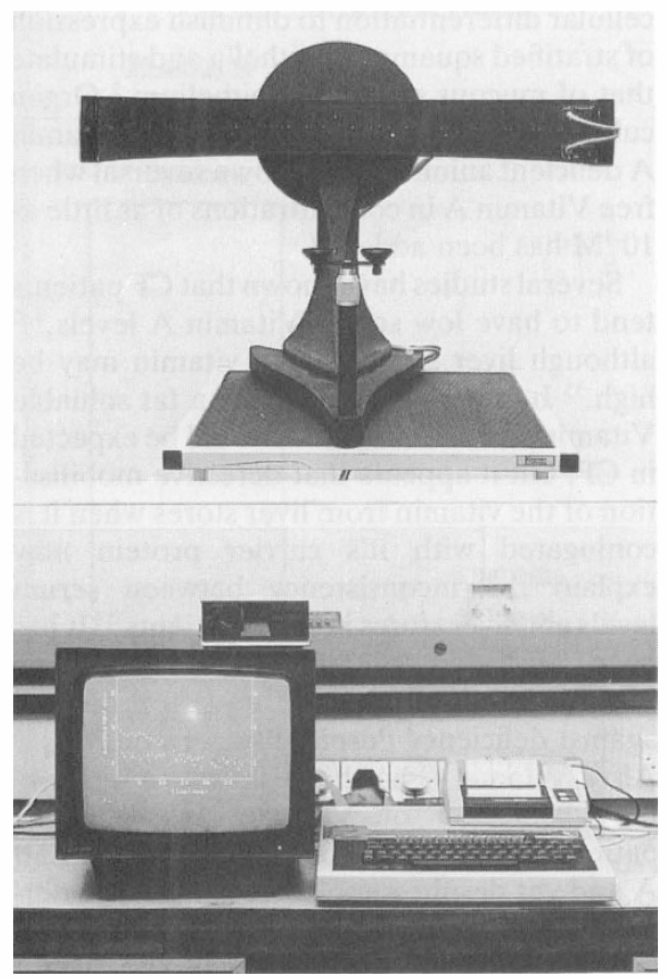

Fig. 1. Photograph showing perimeter with Light Emitting Diodes mounted and the BBC Computer.

puterised system used a BBC microcomputer to control the intensity of the target stimuli, green and red light emitting diodes (LEDs), with peak wavelengths of $555 \mathrm{~nm}$ and $660 \mathrm{~nm}$, which are mounted on a modified perimeter arm. Computer controlled LED dark adaptometers similar to the one used in this study have been shown to give results which correlate with conventional adaptometers such as the Tubingen, ${ }^{9}$ automation simplifying the collection and analysis of data.

In our system, the subject first views a preadapting white screen for 5 minutes with non dilated pupils (screen illumination 2000 $\mathrm{Cd} / \mathrm{sqm}$ ). The perimeter arm has a central dim fixation target and pairs of diodes at 10 and 20 degrees from fixation on the horizontal meridian in the nasal field. A sub-threshold stimulus of $0.5 \mathrm{sec}$ duration is presented to one of the diodes and the intensity increased in $0.1 \mathrm{log}$ unit steps until the subject presses a response button. The computer records this threshold and then tests thresholds at the other three LEDs. This cycle of testing is repeated for 30 minutes allowing the dark adaptation curves to be printed.

For the purposes of this study, we defined abnormal dark adaption to be present if the final threshold to all four targets was more than two standard deviations above the age dependent mean of a control group of 28 subjects. Figure 2 shows the final thresholds for the green target at $20^{\circ}$ from fixation of this normal group, with similar results being obtained at $10^{\circ}$ and for the two red targets.

Blood was taken at routine CF clinic visits-all within three months of the ophthalmological assessment. Liver function was assessed by estimating serum levels of bilirubin, albumin, gammaglutamyltranspeptidase, alanine transaminase and alkaline phosphatase. If any of these were abnormal, the patient was designated as having abnormal liver function.

Vitamin A was measured in batches using High Performance Liquid Chromatography after storage at $-20^{\circ} \mathrm{C}$.

A clinical scoring system ${ }^{10}$ was used to give a numerical indicator of the severity of the CF (Shwachmann Score, scale 100-0, $100=$ excellent).

\section{Results}

Of 52 patients given appointments, 31 were able to attend, (age range 8-44 years, mean age 16). One 8 year old was included in the

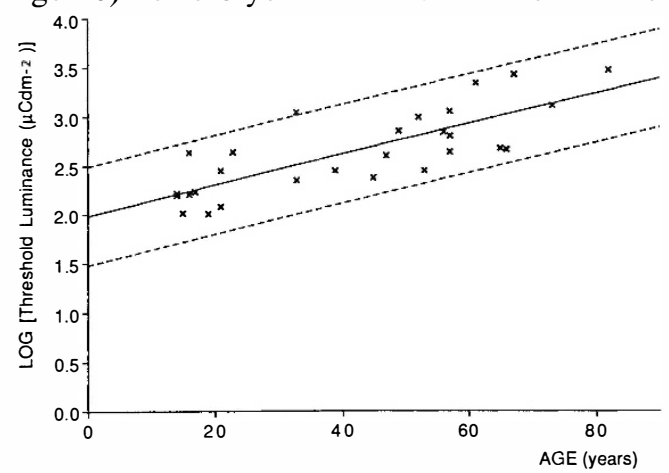

Fig. 2. Dark-adapted thresholds as a function of age for 28 normal subjects (age range 14-82), using green LED stimulus at 20 degrees. The solid line shows a linear fit to the data $(r=0.81)$, broken lines show \pm 2 standard errors. Patients results were classified as abnormal if the thresholds for all four stimuli exceed the age dependent mean by more than two standard errors. 


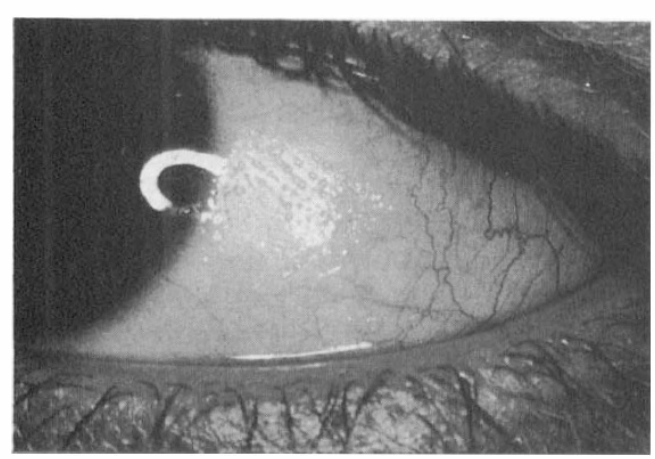

Fig. 3. Macro photograph of eye showing an area of temporal conjunctival xerosis. The appearance is like ripples in the sand at receding tide.

study as he was able to attend with his older brother. None were aware of any eye symptoms, but $3 / 31$ (10 per cent) had conjunctival xerosis (Fig. 3). 6/31 (19 per cent) had abnormal dark adaptation thresholds using the computer system, although two of these patients had normal thresholds determined with the Friedmann. None of the patients had abnormal fundi.

There was no correlation betweeen abnormal dark adaptation and Shwachmann score. ${ }^{4}$

Chi-squared test analysis of dark adaptation and abnormal liver function was not significant, $(\mathrm{p}<0.5)$, (Fig. 5).

Considering Vitamin A levels, on ' $t$ ' test analysis, CF patients with levels $<30$ microgm/dL had raised dark adaptation thresholds. Statistical signficance was at the $\mathrm{p}<0.02$ level when compared with CF patients with levels $>30 \mathrm{microgm} / \mathrm{dL}$, and at the $\mathrm{p}<0.01$ level when compared with normal subjects used as dark adaptation controls, (Fig. 6).

\section{Discussion}

In the late 1930 s, a post mortem study on children dying from CF revealed evidence of Vitamin A deficiency in 10/49 (20 per cent) of cases, deficiency being defined as squamous metaplasia of the bronchial epithelium. ${ }^{3}$ It is interesting that fifty years later nearly 20 per cent of our patients showed evidence of clinical deficiency of the vitamin on ophthalmological grounds despite prescribed supplements.

In epithelial cell culture, Vitamin A directs cellular differentiation to diminish expression of stratified squamous epithelia and stimulate that of mucous secreting epithelium..$^{2}$ Organ cultures of keratinised tracheas from Vitamin A deficient animals have shown reversal when free Vitamin A in concentrations of as little as $10^{-9} \mathrm{M}$ has been added. ${ }^{11}$

Several studies have shown that CF patients tend to have low serum Vitamin A levels, ${ }^{4,12}$ although liver stores of the vitamin may be high. ${ }^{13}$ Impaired absorption of a fat soluable Vitamin such as Vitamin A would be expected in $C F$, but it appears that defective mobilisation of the vitamin from liver stores when it is conjugated with it's carrier protein may explain the inconsistency between serum levels and liver stores in some patients. ${ }^{14}$ It has been suggested that if patients are taking Vitamin A supplements, they are protected against deficiency despite low serum levels. ${ }^{15}$ Also, Vitamin $\mathrm{E}$ has been shown to increase the absorption of Vitamin A. ${ }^{16}$ All our patients had been prescribed Vitamins E and $A$ and yet despite supplementation, a significant percentage had abnormal dark adaptation thresholds or conjunctival xerosis. These patients were not considered to be at particular risk of poor compliance and this may indicate that present supplementation is inadequate in some patients with $\mathrm{CF}$, despite 'normal' liver function.

It has been suggested recently that 'all patients with hepatic involvement in CF should be on Vitamin A supplements. ${ }^{6}$ In our study, no correlation was found between clinical deficiency and abnormal liver function or

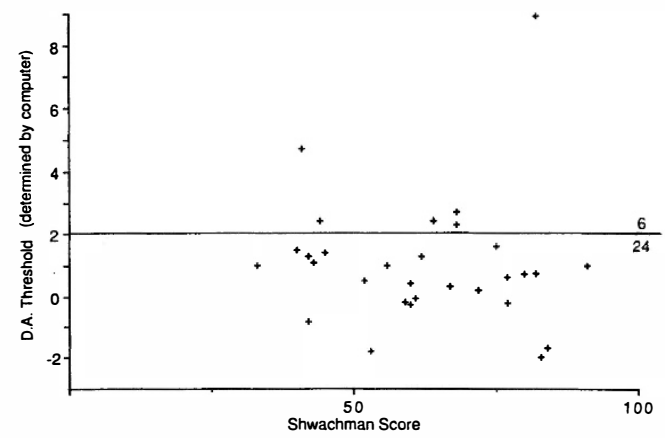

Fig. 4. Graph to show Shwachmann Score plotted against elevations of dark adaptation log threshold (determined by the computer) in standard deviations from the mean of the control group. 


\section{LIVER FUNCTION TESTS}

\section{5 patients}

18 Normal L.F.T.

6 Abnormal

1 Unknown

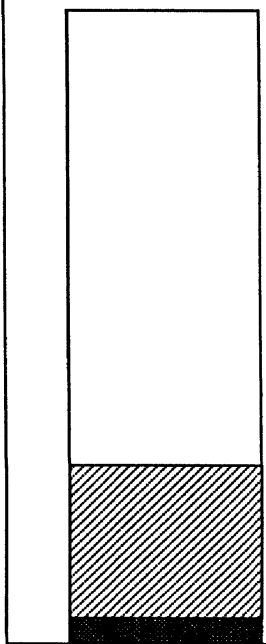

Normal dark adaption
6 patients

3 Normal

3 Abnormal

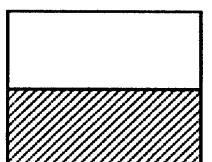

Abnormal dark adaption

Fig. 5. Histogram of liver function status in normal and abnormal dark adaptation groups.

low Shwachmann score, the only risk factor being a Vitamin A level below $30 \mathrm{microgm} /$ dL.

We are only aware of one previous study in which serum Vitamin A and dark adaptation thresholds have been compared in patients with CF. ${ }^{4}$ In this group of 56 patients, details of Vitamin supplementation were not

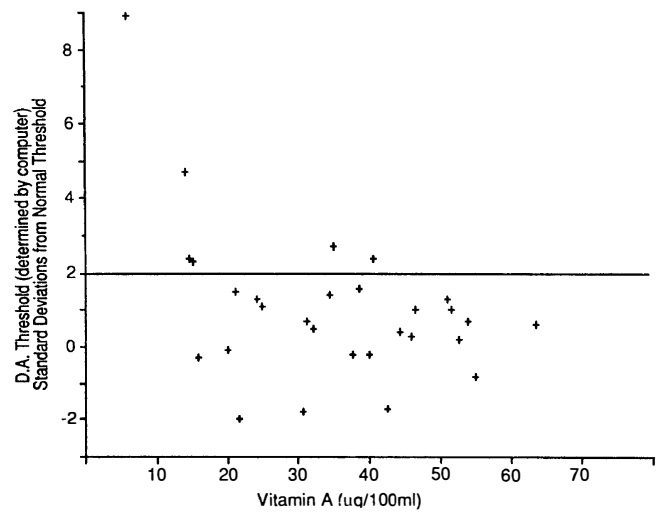

Fig. 6. Graph to show serum Vitamin A levels plotted against elevations of dark adaptation log threshold (determined by the computer) in standard deviations from the mean of the control group.

recorded and no cases of conjunctival xerosis were identified, although it is not clear if slit lamp examination was performed. Two thirds of the group had abnormal liver function and most had a Shwachmann score below 55 (moderate to severe disease). Six patients (10.7 per cent) had dark adaptation thresholds elevated two standard deviations above the normal mean. Although 6/31 (19 per cent) of our patients had abnormal dark adaption thresholds, our findings do not differ significantly on Chi squared analysis from those of the above report.

In 29/31 patients the dark adaptometry results from the two systems correlated but we preferred the computerised system over the Friedmann in our group of young patients. The Friedmann test routine involves informing the patient that a series of three test stimuli are to be displayed. Unfortunately, the test stimulus is accompanied by a click and some of the younger patients gave inconsistent responses whilst they had the audible cue.

These patients were, however, able to give consistent responses with the computerised system and this could explain the contradictory results found between the two systems on two patients. Also, the Friedmann tests the ability to detect a diffuse retinal response whilst the computerised system tests retinal sensitivity at a single point, and this difference may be relevant in these two cases.

In conclusion, we have demonstrated a clinical deficiency of Vitamin A in a proportion of CF patients who have been prescribed the recommended daily pancreatic enzyme and vitamin supplements and have found no correlation between systemic CF status and deficiency of the vitamin.

More CF patients are now surviving to adulthood, receive a mobility allowance and are driving. These patients may be unaware of poor night vision and thus be a hazard to themselves and others.

We would therefore advocate routine Vitamin A supplementation in CF with serum Vitamin A evaluation at regular intervals. Ophthalmological assessment is indicated for patients with Vitamin A levels below 30 microgm/dL to identify those who have clinical deficiency. 
We would like to thank Beverley Hudson and Elaine Franklyn for their technical assistance. Dr Tyrrell's post was funded by The Cystic Fibrosis Research Trust.

\section{References}

${ }^{1}$ Prof J. Dodge: Chairman British Paediatric Association Cystic Fibrosis Working Party-Personal Communication.

2 Wolf G: Multiple Functions of Vitamin A. Physiol Rev 1984, 64: 873-937.

${ }^{3}$ Andersen DH: Cystic Fibrosis of the Pancreas, Vitamin A deficiency, and Bronchiectasis. J Pediat 1939, 15: 763-71.

${ }^{4}$ Fulton AB, Hansen RM, Underwood BA et al.: Scotopic Thresholds and Plasma Retinol in Cystic Fibrosis. Invest Ophthalmol Vis Sci 1982, 23: 364 70.

${ }^{5}$ Kulzycki LL: Malabsorption with Vitamin A deficiency in a college girl treated for Cystic Fibrosis. Acta Paediatr Scand 1971, 60: 371-2.

${ }^{6}$ O'Donnell $\mathrm{M}$ and Talbot JF: Vitamin A deficiency in treated Cystic Fibrosis: case report. $\mathrm{Br} J$ Ophthalmol 1987, 71: 787-90.

${ }^{7}$ Petersen RA, Petersen VS, Robb RM: Vitamin A deficiency with Xerophthalmia and night blindness in Cystic Fibrosis. Am J Dis Child 1968, 116: $662-5$.

${ }^{8}$ Friedmann Analyser Instruction Manual Clement Clarke Ltd London.
${ }^{9}$ Ernst W, Falkener DJ, Hogg CR et al.: An automated static perimeter/adaptometer using light emitting diodes. Br J Ophthalmol 1983, 67: 43142.

${ }^{10}$ Shwachmann $\mathrm{H}$ and Kulczycki LL: Long term study of one hundred and five patients with Cystic Fibrosis. Studies made over a five to fourteen year period. Am J Dis Child 1958, 96: 6-15.

${ }^{11}$ Sporn MB, Clamon GH, Dunlop NM et al.: Activity of Vitamin A analogues in cell cultures of mouse epidermis and organ cultures of hamster trachea. Nature 1975, 252: 47-50.

${ }^{12}$ Congden PJ, Bruce G, Rothburn NM et al.: Vitamin status in treated patients with Cystic Fibrosis. Arch Dis Child 1981, 56: 706-14.

${ }^{13}$ Underwood BA and Denning CR: Blood and liver concentrations of Vitamin $\mathrm{A}$ and $\mathrm{E}$ in children with Cystic Fibrosis of the Pancreas. Pediatr Res 1972, 6: 26-31.

${ }^{14}$ Rasmussen M, Michalsen H, Lie SO et al.: Intestinal retinaol esterification and serum retinol in children with Cystic Fibrosis. J Pediatr Gastroenterol Nutr 1968, 5: 397-403.

15 Underwood BA: The determination of Vitamin A and some aspects of it's distribution, mobilisation and transport in health and disease. World Rev Nutr Diet 1974, 19: 123-72.

${ }^{16}$ Ekvall S and Mitchell A: The effect of supplemental Vitamin E on Vitamin A serum levels in Cystic Fibrosis. Int J Vitam Nutr Res 1978, 48: 324-32. 